\title{
Uso del tiempo e interacciones en la sala de clases. Un estudio de casos en Chile ${ }^{1}$
}

Sergio Martinic*, Claudia Vergara** , David Huepe***

\section{Resumen}

Este artículo analiza las interacciones en la sala de clases y el uso del tiempo de profesores de segundo ciclo de Enseñanza Básica y que han participado en el Sistema de Evaluación de Desempeño Profesional que ejecuta el Ministerio de Educación de Chile. Se demuestra que las clases son altamente estructuradas y se observa un claro patrón de interacción y uso del tiempo en las clases video grabadas de los profesores considerados. El discurso de los profesores ocupa el $51 \%$ del tiempo de la clase y su contenido está centrado en instrucciones o procedimientos de trabajo y exposición de la materia. Las intervenciones verbales de los alumnos ocupan muy poco tiempo y sus acciones respon-

* Profesor Asociado Pontificia Universidad Católica de Chile. smartini@uc.cl

* * Profesora Universidad Católica Silva Henríquez, Chile. cvergara@ucsh.cl

* * * Profesor Universidad Diego Portales, Santiago, Chile. david.huepe@mail.udp.cl den y están claramente enmarcadas por los profesores.

\section{Palavras-chave}

interacción profesores y alumnos; uso del tiempo; patrones instruccionales.

1. Articulo elaborado en el marco de los proyectos Interacciones pedagógicas y uso del tiempo en el aula, Proyecto CIE01-CONICYT, Pontificia Universidad Católica de Chile y FONDECYT № 1110601 Tiempo escolar, intercambios y exigencia cognitiva en aulas de clase con alto y bajo Simce en el segundo ciclo de Enseñanza Básica. 


\title{
The use of time and interactions in the classroom. A case study in Chile
}

\begin{abstract}
This article discusses the interactions in the classroom and the use of time of teachers from the second cycle of Basic Education who have participated in the Professional Performance Evaluation System conducted by the Ministry of Education in Chile. The video recordings reveal that the classes are highly structured and a clear pattern of interaction and use of time in class can be observed. The teachers' talking time occupies $51 \%$ of the lesson and the content of their discourse is focused on task instructions or procedures and presentation of the subject matter. The students' verbal interventions take very little time and their actions are clearly framed and guided by the teachers.
\end{abstract}

Key words teachers and students' interaction; use of time; instructional patterns. 


\section{Antecedentes}

En los últimos años los estudiantes chilenos han mejorado sus aprendizajes. Una demostración de ello son los resultados de la prueba PISA del año 2009 y los resultados de la Medición Simce del 2010 en los cuales se constata, particularmente, un aumento en los aprendizajes de lectura ${ }^{2}$. Pese a ello el promedio general de los resultados está por debajo de los promedios internacionales y se constatan grandes desigualdades en su distribución (Chile, 2010a).

La situación afecta, particularmente, a los alumnos más pobres y que asisten a las escuelas públicas gestionadas por los municipios. En las mediciones del Simce un alumno que asiste a una escuela municipal y que pertenece a familias vulnerables puede tener 80 puntos menos en matemáticas que un niño que asiste a un establecimiento privado y que pertenece a familias del sector medio o alto (Chile, 2009, 2010 b).

Las explicaciones del problema son muchas y la investigación educacional demuestra que estas pueden agruparse en dos grandes categorías. La primera de ellas subraya la importancia de la familia y de su capital cultural (Coleman, 1988; Unicef, 2005); la segunda pone atención en los procesos internos de las escuelas particularmente en la gestión directiva y el desempeño pedagógico de los profesores en la sala de clases (Martinic et al., 2003; Murillo, 2003; Arrau, 2004; Brunner et al., 2006).

El capital cultural de la familia y sus expectativas educacionales tienen una alta incidencia en los resultados de aprendizaje. Sin embargo, los estudios demuestran que, una vez controlados los factores culturales asociados a la familia los resultados dependen centralmente de lo que ocurre en las escuelas en la interacción entre sus profesores y en las prácticas pedagógicas en la sala de clases.

En efecto, son las oportunidades educativas y las situaciones pedagógicas las que favorecen el desarrollo y aprendizaje de los estudiantes. Desde esta perspectiva, la interacción pedagógica en la sala de clases; la pertinencia y relevancia de contenidos curriculares; el uso del tiempo; la disciplina y el clima de aula que genera el profesor tienen una fuerte incidencia, en la efectividad de las escuelas y en el logro de mejores aprendizajes.

Al interior de estos procesos pedagógicos la organización del tiempo escolar y la gestión del tiempo en la sala de clases aparecen como importantes factores que inciden en

2. Sistema Nacional de Medición de la Calidad de la Educación (SIMCE). www.simce.cl 
los resultados de los aprendizajes, particularmente en los sectores de pobreza (Russell, 2001; Dodd, 2002; Metzker, 2003; Strasser, Rosa, Silva e Silva, 2009).

Por ello, la ampliación y uso del tiempo pedagógico suele ser objeto de políticas para el mejoramiento de las prácticas. Es el caso de Chile que, desde 1997, implementa la Jornada Escolar Completa (JEC). La JEC nace con el objetivo de ampliar y reestructurar el uso del tiempo en las escuelas para ponerlo al servicio del mejoramiento de los aprendizajes y de la innovación pedagógica en las escuelas subvencionadas (Cox, 2005; Brunner et al., 2006; Valenzuela, 2006)³.

\section{El problema}

Pese a la fuerza de esta relación, la investigación demuestra que los resultados de aprendizaje no dependen exclusivamente del tiempo asignado. El tiempo escolar puede ser representado como una pirámide invertida. En la base superior se encuentran las unidades macro de tiempo (total de días y horas de clases en el año escolar), en el centro el tiempo asignado a actividades curriculares y en el vértice invertido de la pirámide el tiempo instruccional y comprometido para el aprendizaje en la sala de clases (Metzker, 2003).

En el caso de Chile, se ha demostrado que la ampliación del tiempo escolar ha tenido un impacto modesto en el tiempo instruccional y en los aprendizajes. La ampliación de horas de clases o de la Jornada Escolar (JEC) ha significado para los establecimientos un incremento en los aprendizajes de 1.6 puntos en matemáticas y 1.8 puntos en el sector de Lenguaje anuales (Chile, 2002). Resultados similares se confirman en Valenzuela (2005) y Bellei (2006) y en un estudio del Simce en el que se constata que los establecimientos con más de 5 años en JEC tienen una mejoría en 5 puntos en promedio (Chile, 2009) 4

Existe una tensión entre el concepto de tiempo que opera en la práctica y los requerimientos que demanda el sistema para mejorar los resultados de aprendizaje.

3. Con esta política la jornada escolar aumenta las horas de clases en un $30 \%$ en la Enseñanza Básica y Media. Con ello, el país alcanza un total de 1.100 horas anuales cronológicas de docencia desde $3^{0}$ a $8^{0}$ básico y de 1.216 horas en Educación Media superando el promedio de horas de los países de la OECD quienes disponen para un programa curricular típico a los 14 años de 944 horas de instrucción.

4. SIMCE. Sistema de Medición de la Calidad de la Educación. www.simce.cl
Hoy día está en discusión el concepto objetivo y absoluto de tiempo y que atribuye al agregar o quitar horas de clases un incremento proporcional y directo en el aprendizaje (Husti, 1992; Sue, 2005; Metzker, 2003).

En efecto, en la investigación reciente, el tiempo se entiende como una variable dependiente 
asociada a la interacción y participación activa de los sujetos en la situación de aprendizaje. Los estudios demuestran que el mayor tiempo de la jornada escolar genera mejores aprendizajes siempre y cuando sea acompañado de tiempo efectivo dedicado a la tarea por parte de los estudiantes, nuevas prácticas y de un reordenamiento de los medios didácticos, de las estructuras curriculares y, de los contenidos educativos que se enseñan (Metzker, 2003; KARAMPELAS, 2005).

Desde esta perspectiva no basta con aumentar horas de enseñanza para mejorar los aprendizajes sino que, por el contrario, lo relevante es el tiempo invertido por los estudiantes en la tarea y ello es variable de acuerdo a los sujetos, sus capacidades, ritmos y calidad de la interacción pedagógica (Delhaxhe, 1997; Karampelas, 2005; Wubbels, Brekelmans, 2005; Bellei, 2006).

Asumiendo esta perspectiva llevamos a cabo una investigación que aborda las interacciones en la sala de clases y el uso o gestión del tiempo que realizan profesores de segundo ciclo de Enseñanza Básica y de primer ciclo de Enseñanza Media y que han participado en el Sistema de Evaluación de Desempeño Profesional que promueve el Ministerio de Educación5. El objeto del estudio son las interacciones pedagógicas en la sala de clases y su relación con el uso o gestión del tiempo. Al mismo tiempo se relacionará las interacciones y la gestión del tiempo con distintos niveles de complejidad y de exigencia cognitiva ${ }^{6}$.

\section{Material y método de análisis}

El material analizado corresponde a 29 videos en los que se registran las clases presentadas por profesores de Educación Básica y Media de los sectores de Lenguaje y Matemáticas. Estas clases han sido grabadas en el marco de la evaluación de desempeño docente implementada por el Ministerio de Educación y cada una de ellas tiene una duración de 40 minutos.

Las clases son analizadas como una interacción comunicativa y para la descripción y análisis se optó por el Análisis Conversacional. Se trata de un método cuyo objeto es la descripción e interpretación de los procedimientos que los sujetos emplean para producir organizada y coordinadamente un discurso en una situación comunicativa (Candela, 2001; Moeschler, 1989; Schiffrin, 1994).

5. Es una evaluación regulada por ley y obligatoria para los profesores que se desempeñan en establecimientos del sistema público o municipal. Entre los materiales del portafolio solicitado a los profesores se encuentra un video de una clase. Más información del sistema de evaluación en www.docentemas.cl

6. La investigación está en curso y se realiza en el marco del proyecto FONDECYT № 1110601 Tiempo escolar, intercambios y exigencia cognitiva en aulas de clase con alto y bajo Simce en el segundo ciclo de Enseñanza Básica. 
Para el análisis de la conversación el discurso es resultado del diálogo y de la influencia recíproca de los locutores. Por ello, la unidad de estudio es el intercambio y que da cuenta de la situación de interacción verbal y del juego de réplica que este implica.

Los intercambios constituyen las unidades dialógicas más pequeñas de cualquier conversación (Roulet, 1985; Moeschler, 1989). Cada intercambio se compone, al menos, de dos turnos de palabra de locutores diferentes. Cada uno de estos turnos constituye una intervención, las que realizan actos de lenguaje. Por ejemplo, una intervención inicial del Profesor es seguida por una intervención reactiva del Alumno la que, posteriormente, es evaluada por otra intervención del mismo profesor. De este modo el intercambio queda compuesto por tres tipos de intervenciones: Inicial, Reactiva y Evaluativa (IRE) y que corresponden a cada uno de los movimientos anteriormente descritos. Los intercambios, a su vez, se integran en unidades mayores denominadas secuencias y que agrupan intercambios relacionados fuertemente por su coherencia semántica o pragmática (Roulet, 1985; Kerbrat-Orechioni, 1998).

En las condiciones reales de una sala de clase la interacción del profesor con sus alumnos es continua. Por ello, y para aproximarse a esta situación, se ha optado por un procedimiento de observación continuo, clasificando las intervenciones de los profesores y alumnos cada 4 segundos durante toda la clase. Este procedimiento permite tener una medida exacta de la duración y de la frecuencia de cada una de las intervenciones (Croll, 2000). A través de este procedimiento es posible saber el número de veces que se realiza un acto específico; la proporción de tiempo que ocupa, el lugar de su realización y las acciones que la preceden o las que se desarrollan posteriormente. Se ocupó el videograph para la clasificación de los videos en función de las categorías definidas (Koch, Sabine, Zumbach, 2002). En este artículo se analizan aspectos relacionados con las intervenciones de profesores y alumnos.

\section{Resultados}

\section{Organización social de la clase}

La clase tienen una organización y que resulta de la distinción de fases o segmentos denominados inicio, desarrollo y cierre (NCES,2003; Forero, 2008). La distinción entre ellos se realiza a través de "marcadores" lingüísticos, interaccionales o gestuales y que permiten transitar claramente de una fase a otra. 
El inicio y el cierre definen el tiempo total empleado en la clase. En las fases de inicio y cierre es conveniente distinguir, como sugiere el estudio del Timss (2002), un momento de pre inicio y otro de preparación del cierre y que permiten dar cuenta de actividades particulares de transición. Del mismo modo, al interior de la fase de desarrollo se distinguen secuencias de acuerdo a la unidad temática o tipo de actividad que se desarrolla en el tratamiento de la materia.

Las clases observadas dedican, en promedio, un total de 7.2 minutos para el pre inicio e inicio de la clase propiamente tal, lo que corresponde al 18,2\% del tiempo total de la clase. La fase de desarrollo ocupa 28,4 minutos representando el $71,2 \%$ del tiempo total de la clase. Llama la atención que la preparación del y el cierre propiamente tal ocupen en promedio 4,2 minutos lo que equivale al 10,6\% del tiempo de la clase. Ello da cuenta de un problema de gestión ya que, pese a ser clases registradas en el contexto de evaluación docente, muchos profesores no anticipan un cierre pedagógico adecuado.

\section{Tiempo y habla en profesores y alumnos}

En el conjunto de las clases observadas el profesor ocupa la mayor parte del tiempo (51\%) y, por lo general, dirige su habla a la clase como un todo $(44,7 \%$ del tiempo), dedicándose a exponer materias o explicar procedimientos. El estilo de enseñanza que predomina no produce grandes diferencias entre los alumnos constituyendo al grupo curso en un "alumno promedio".

En segundo lugar, destaca el tiempo clasificado como silencio $(19,8 \%$ ) y que corresponde a la realización de actividades por parte de los alumnos y que responden a instrucciones o enmarcamiento realizado por el profesor. Por último, y en un $14,2 \%$ del tiempo de la clase, se observa a alumnos solos realizando intervenciones que responden, principalmente, a preguntas e instrucciones del profesor. En este caso las intervenciones tienen diferencias importantes según el sector de aprendizaje. En el sector de lenguaje los alumnos tienen más tiempo de intervención verbal.

En términos generales se constata un estilo de enseñanza en el cual predomina una pedagogía directiva con poco diálogo y conexión con los estudiantes tal como ha sido demostrado en otros estudios (Martinic, Vergara, 2007).

La participación y el protagonismo que tienen alumnos y profesores cambian a medida que transcurre la clase. En los gráficos 1 y 2 se representan el tiempo que ocupan las intervenciones de profesores y alumnos durante la clase. 
Gráfico 1 - Hablante principal Profesor (Matemáticas y Lenguaje)

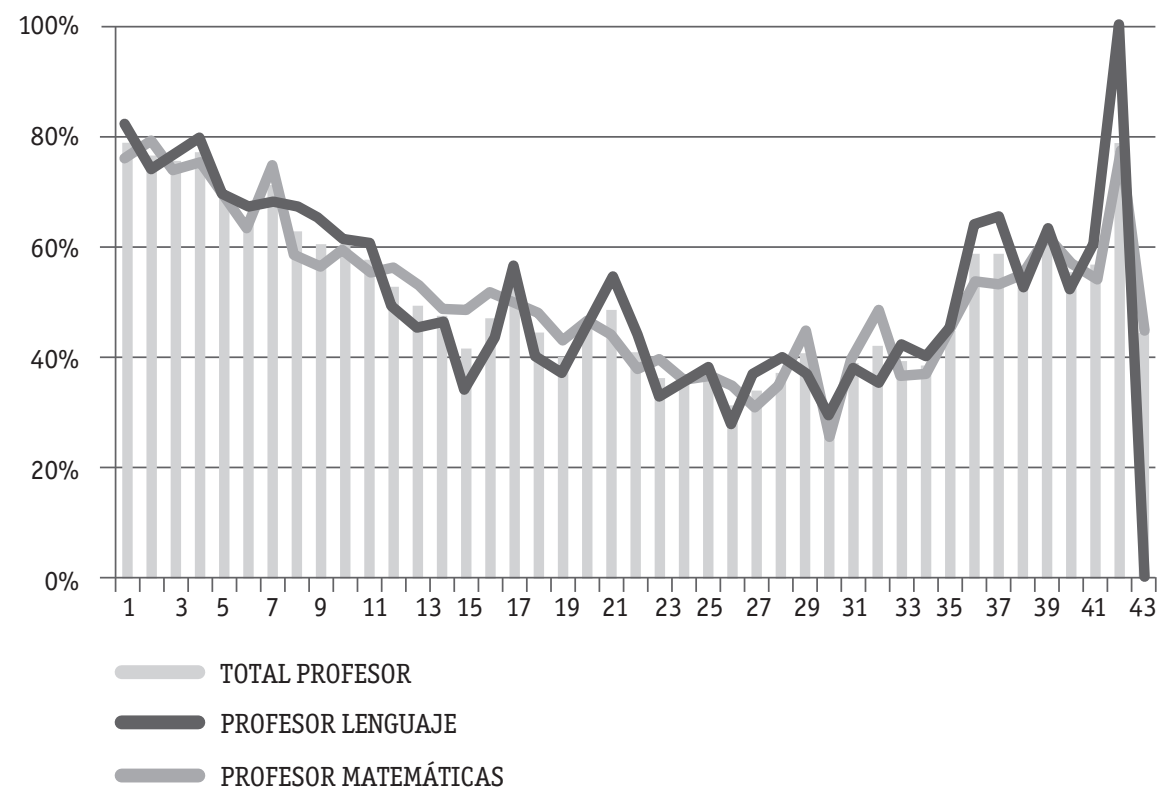

Gráfico 2 - Hablante principal un Alumno en Lenguaje y Matemáticas

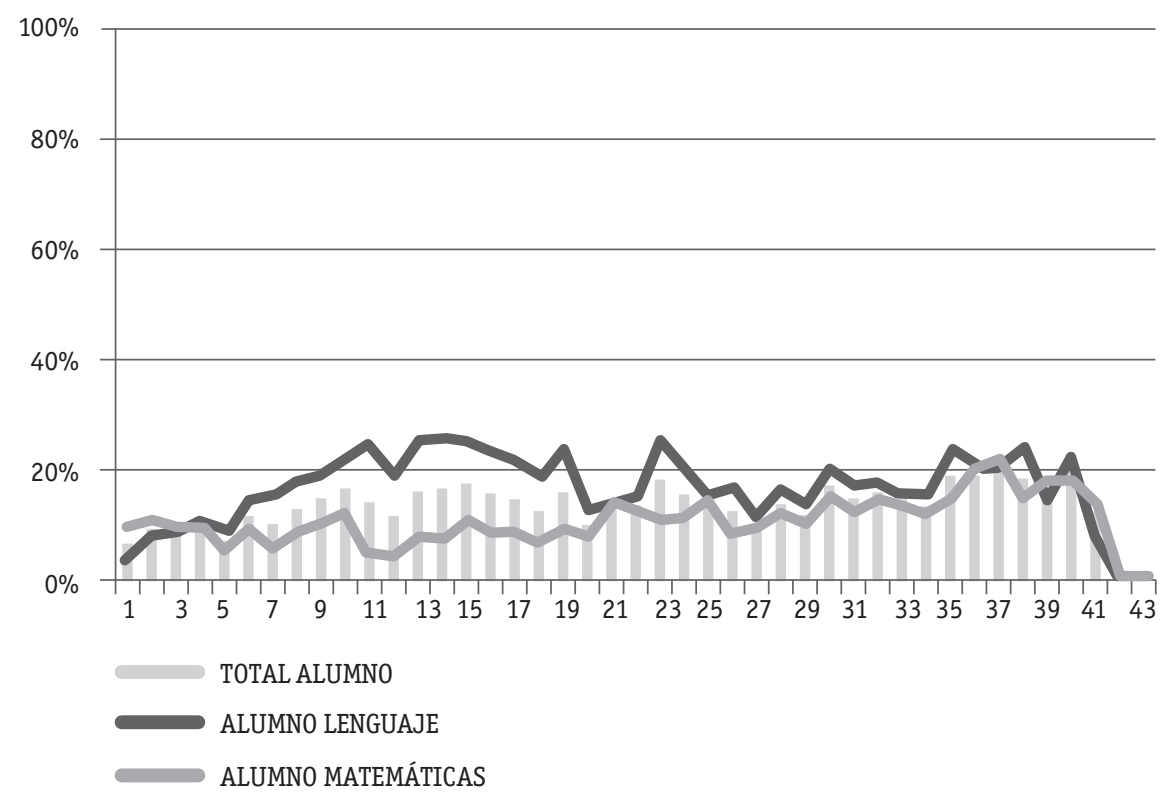


En promedio el habla del profesor ocupa la mayor parte del tiempo de la clase. Sin embargo ocurren movimientos importantes en el transcurso de la clase. Como se aprecia en los gráficos los profesores ocupan la mayor parte del tiempo los primeros siete minutos para luego bajar a la mitad del tiempo entre los minutos 15 y 19 y en los últimos cinco minutos vuelve a tener un aumento en la fase de preparación y de cierre propiamente tal. Se observan interesantes diferencias entre los profesores de lenguaje y de matemáticas. En promedio las intervenciones de los profesores de matemáticas ocupan más tiempo que los de lenguaje. Solo en el minuto 27 de la clase disminuyen sus intervenciones para que aumenten las de los alumnos recuperando en el minuto 32 la palabra con bastante protagonismo.

Este perfil del tiempo que ocupa el profesor se distingue claramente de la intervención de los alumnos. Ellos adquieren mayor protagonismo en el momento del desarrollo de la clase (minutos 21-23) y en la fase de preparación del cierre (minutos 34 a 37). La participación de alumnos tiene más importancia en términos de uso del tiempo en las clases de lenguaje que en las de matemáticas.

\section{Tipo de intervención de profesores y alumnos}

Al analizar el tipo de intervención de los profesores de Lenguaje y Matemáticas se observa que la mayor parte del tiempo se concentra en instrucciones o información relacionada con procedimientos a seguir. La exposición de contenidos de la disciplina propiamente tal ocupa el 9,5\% del tiempo de habla de los profesores de lenguaje y el 9,1\% del tiempo de habla de los profesores de matemáticas.

En cuanto a las intervenciones de los alumnos se observa que la mayor parte del tiempo se dedica a realizar tarea o a trabajar en función de actividades instruidas por los profesores. El 14,1\% del tiempo de las intervenciones de los alumnos en lenguaje aluden a respuestas a preguntas del profesor. En matemáticas este alcanza el 13,4\% del tiempo de sus intervenciones. En ambos casos predominan respuestas con baja elaboración donde los alumnos se limitan a repetir o aprobar afirmaciones y contenidos enunciados por el profesor. Son preguntas que desarrollan intercambios de baja complejidad cognitiva en los cuales los alumnos no actúan sobre el objeto de conocimiento ni sus interacciones provocan cambios en el ambiente físico y social de la clase (Mares et al., 2005; Martinic, Vergara, 2007).

En síntesis, las interacciones observadas son altamente estructuradas por el profesor y en las que los alumnos tienen un rol pasivo con intervenciones que ocupan poco tiempo en el conjunto de la clase. En cuanto al tipo de intervención, predomina en los profesores el tiempo invertido en preguntas, las que son principalmente de 
baja complejidad cognitiva (Martinic; Vergara, 2007). Estos resultados son consistentes con los que han encontrado González, Preiss, San Martin (2008) y Preiss (2009) en los que las preguntas y evaluaciones de los profesores están centradas, más bien, en el control del flujo de la clase y verificación de información.

\section{Conclusiones}

El análisis demuestra que se produce una convergencia de los casos en determinados patrones de interacción y de uso del tiempo. Este es altamente estructurado, en el cual los profesores exponen, los estudiantes aplican y los profesores controlan dicha aplicación en el transcurso de la clase. Se observa que el rol docente y la organización didáctica en la sala de clase no ha cambiado sustancialmente en los últimos años y el protagonismo del profesor y la transmisión directiva de contenidos sigue ocupando la mayor frecuencia de tiempo (Filp, 1987; Villalta, 2003).

Diversos estudios demuestran que estas interacciones son frecuentes en las escuelas que trabajan con niños provenientes de los sectores más pobres de la sociedad (De La Cruz et al., 2001, Martinic, 2006; Draelents, 2009). Por lo general, los docentes de escuelas periféricas acentúan los aspectos disciplinarios por sobre los cognitivos, y en el aula ponen énfasis en el control del comportamiento de sus alumnos.

Estos patrones de interacción y de uso del tiempo pueden explicarse por varios factores. Entre ellos, el contexto de evaluación en el cual se realizaron los registros; las políticas públicas, el tamaño del curso; el tipo de dependencia; el curriculum, los textos escolares, entre otros.

Por último, una explicación de naturaleza cultural. Como lo demuestra el estudio de observación de clases realizado por el Timss (Bogard, et al, 2005) los profesores tienden a reproducir la práctica de enseñanza que vivieron como estudiantes en la escuela. La organización de la clase, la estructura en el tiempo y la interacción comunicativa reproduce prácticas de su propia experiencia escolar.

Sin embargo, es importante señalar que la convergencia se da en categorías macros. Por ejemplo, en la estructura de la clase; dirección del habla, entre otras. Al analizar con más detalle las intervenciones de profesores y alumnos se observan diferencias importantes. Por ello, es necesario pasar de categorías macro a micro para dar cuenta de la complejidad de las interacciones situadas. El análisis de los intercambios ofrece un análisis más complejo y que da cuenta del efecto de interacción y de la complejidad cognitiva en la gestión del tiempo. 


\section{Referencias bibliográficas}

ARRAU, A. Bases para la competencia en Chile. La educación en una sociedad desigual. Santiago: Ril, 2004.

BELLË̈, C. Does Lengthening the School Day Increase Students' Academic Achievement? Results from a Natural Experiment in Chile. Harvard: Harvard Graduate School, 2006.

BOGARD, K.J.; HIEBERT, J.; HOLLINGSWORTH, H.V; GALLIMORE, R. Are there national patterns of teaching ? Evidence from the TIMSS 1999 video study. Comparative Education Review, v. 49, n. 3, p. 311-343, 2005.

BRUNNER, J.J.; ELACQUA, G. Calidad de la educación. Claves para el debate. Santiago: Ril, 2006.

CANDELA, A. Corrientes teóricas sobre discurso en el aula. Revista Mexicana de Investigación Educativa, México, v. 6, n. 12, p. 317-333, 2001.

CHILE. Ministerio de Educación. División de Planificación y Presupuesto. Prueba SIMCE 2ํo medio 2001. Análisis de resultados. Santiago: Ministerio de Educacion de Chile. 2002.

CHILE. Ministerio de Educación. Resultados Nacionales, Simce, 2008. Santiago: Mineduc, 2009.

CHILE. Ministerio de Educación. Resultados Nacionales, Simce, 2009. Santiago: Mineduc, $2010 a$.

CHILE. Ministerio de Educación. Resumen de resultados PISA 2009-Chile. Santiago: Simce, 2010b.

COLEMAN, James. Social capital in the creation of human capital. American journal of sociology, v. 94, p. 95-120. 2008. Disponible en: http://www.jstor.org/stable/10.2307/2780243. Acceso 15 febrero 2013.

COX, Cristián. (ed). Políticas educacionales en el cambio de siglo. La reforma del sitema escolar chileno. Santiago: Editorial Universitaria.2005.

CROLL, P. Systematic Classroom Observation. London: Routledge Falme, 2000.

DE LA CRUZ, M.; SCHEUER, N.; CAINO, G.; HUARTE, M.; BAUDINO, V.; AYASTUY, R. El discurso en clase de maestros de nivel primario en distintos sectores socioculturales. Estudios Pedagógicos, Chile, n. 27, p. 2-41, 2001.

DELHAXHE, A. Le temps comme unité d'analyse dans la recherche sur l'enseignement. Revue Francaise de Pédagogie, Paris n. 118, p. 107-125, 1997. 
DODD, C. Extended-Day Programs: Time to learn. Leadership. California, v. 32, n. 1, p. 24-25, sep-oct. 2002.

DRAELENTS, H. Reforme pédagogique et légitimation. Bruxelles: De Boeck, 2009.

FILP, J.; CARDEMIL, C. Disciplina, control social y cambio: estudio de las prácticas pedagógicas en una escuela básica popular. Santiago: CIDE, 1987.

FORERO, A. Interacción y discurso en la clase de matemáticas. Universitas Psychologica, Bogotá, v. 7, n. 3, p. 787-806, 2008.

GONZÁLEZ, J.; PREISS, D.; SAN MARTIN, E. Evaluando el discurso docente: desarrollo de un modelo de Rasch a partir de la evidencia audiovisual de profesores chilenos de primer ciclo de Educación Básica en el área de Lenguaje. Revista Iberoamericana de Evaluación Educativa, n. 1, 2008. Extraído de: 〈http://rinace.net/riee/numeros/vol1-num2/art9.pdf〉. Consultado el 20 nov. 2010.

HUSTI, A. Del tiempo escolar uniforme a la planificación móvil del tiempo. Revista de Educación, n. 298, p. 271-305, 1992.KARAMPELAS, K. Re-designing time management in reponse to educational change. In: INTERNATIONAL CONGRESS FOR SCHOOL EFFECTIVENESS AND IMPROVEMENT, 2005, Barcelona, 2005.

KERBRAT-ORECCHIONI, C. Les interactions verbales. Approche interactionnelle et structure des conversations. 3. ed. Paris: Armand Colin, 1998. Tomo 1.

KOCH, S. C.; SABINE; ZUMBACH, J. The Use of video Analysis Software in Behavior Observation Research: Interaction Patterns in Task-Oriented Small Groups. Qualitative Social Research, v. 3, n. 2, 2002. Extraído de: http://www.qualitative-research.net. Consultado: 4 nov. 2009.

MARES, G.; GUEVARA, E. R.; RIVAS, O.; ROCHA, R. Análisis de las interacciones maestra-alumnos durante la enseñanza de las ciencias naturales en primaria. Revista Mexicana de Investigación Educativa, México, v. 9, n. 22, p. 721-745, 2005.

MARTINIC, S. Representaciones de la desigualdad en la cultura escolar. Revista Persona y Sociedad. Santiago: Universidad Alberto Hurtado-Ilades, v.XVII, n. 1, p.129-146.2003.

MARTINIC, S. El estudio de las representaciones y el análisis estructural del discurso. In: CANALES, M. (Org.). Metodologías de investigación social. Santiago: Lom, 2006. p. 299-320.

MARTINIC, S.; VERGARA, C. Gestión del tiempo e interacción del profesor-alumno en la sala de clases de establecimientos de Jornada Escolar Completa en Chile. Revista Electrónica Iberoamericana sobre Calidad, Eficacia y Cambio en Educación, Madrid, v. 5, n. 5, p. 3-20, 2007.

MURILLO, J. (coord). La investigación sobre eficacia escolar en Iberoamérica. revisión internacional del estado del arte. Bogotá: Convenio Andrés bello.2003 
MAURICE, J-J. ; ALLÉGRE, E. Invariance temporelle des pratiques enseignantes: le temps donné aux éleves pour chercher. Revue Française de Pédagogie, Paris, n. 138, p. 115-124, 2002.

METZKER, B. Time and Learning. 2003. Extraído de: http://searcheric.org/ericdc/ ED474260.htm. Consultado el 20 nov. 2010.

MOESCHLER, J. Marques linguistiques, intérpretation, pragmatique et conversation. Cahiers de Linguistique Française, Genève, n. 10, p. 43-76, 1989.

NCES. Third International Mathematics and Science Study 1999 Video Study. Washington, DC: Department of Education, 2003.

PREISS, D. The Chilean instructional pattern for the teaching of language: a video-survey study based on a national program for the assessment of teaching. Learning and Individual Differences, Amsterdam, v. 19, n. 1, p. 1-11, 2009.

ROULET, E. L'articulation du discours en français contemporain. Berne: Peter Lang, 1985. ROULET, E. Une description modulaire de l'organisation topicale d'un fragment d'entretien. Cahiers de linguistique française, Genève, n. 18, p. 11-32, 1996.

RUSSELL, M. After-school programs are making a difference. National Association of Secondary School Principals Bulletin. Proquest Educational Journals, v. 86, n.626, p. 3-20, 2001.

SCHIFFRIN, D. Approaches to discourse. Oxford: Blacwell, 1994.

STRASSER, K.; ROSA, M.; SILVA, M.; SILVA, L. Y. Time Management in 12 Chilean Kindergarten Classrooms: Recess, Snack and a Little Teaching. Management, v. 18, n. 1, p. 85-96, 2009.

SUE, R. Les temps nouveaux de l'éducation. In: Sue, R.; CACCIA, M. (Org.). Autrestemps, autre école. Impact et enjeux des rythmes scolaires. Paris: Retz, 2005. p. 193-203.

UNICEF ¿Quién dijo que no se puede?- Escuelas efectivas en sectores de pobreza. Santiago: Unicef. 2005.

VALENZUELA, J. P. Partial Evaluation of a Big Reform in the Chilean Education System: FromaHalfDaytoaFullDaySchooling.TesisPh.D.Economía,University ofMichigan.2005

VILLALTA, M. Uso del tiempo en la interacción didáctica de sala de clase. Revista Investigaciones en Educación, v. 3, p. 101-114, 2003.

WUBBELS, T; BREKELMANS, M. Two decades of research on teacher-student relationships in class. International Journal of Educational Research, n. 43, p. 1161-1191, 2005.

Recebido em 3 de abril de 2012 e aprovado em 17 de agosto de 2012. 